\title{
Erratum: Dropping an impurity into a Chern insulator: A polaron view on topological matter [Phys. Rev. B 99, 081105(R) (2019)]
}

\author{
A. Camacho-Guardian (1), N. Goldman, P. Massignan, and G. M. Bruun \\ (Received 1 September 2020; published 24 September 2020)
}

DOI: 10.1103/PhysRevB.102.119903

In the original paper, we studied the transport properties of an impurity coupled to a Chern insulator. Due to a subtle numerical error which we have discovered in our original codes, some of our findings should be revised.

The main conclusion that the transverse drag exerted by the dressing cloud on the impurity exhibits a sharp jump whenever the surrounding cloud enters a topologically nontrivial state remains valid. However, we found the conductivity to be small but nonzero outside of the topological region. Figure 1 should also be revised. The corrected phase diagram is shown in Fig. 1(a) with vertical and horizontal cuts shown in Figs. 1(b) and 1(c).

An interesting new feature is that the transverse conductivity vanishes and changes sign at $\phi= \pm \pi / 2$ due to a particle-hole symmetry. We will analyze this interesting effect in more detail in a forthcoming paper [1].

We are indebted to D. Pimenov and M. Goldstein for alerting us to the possible presence of a numerical error, for valuable discussions, and for assisting us in double-checking our numerics.

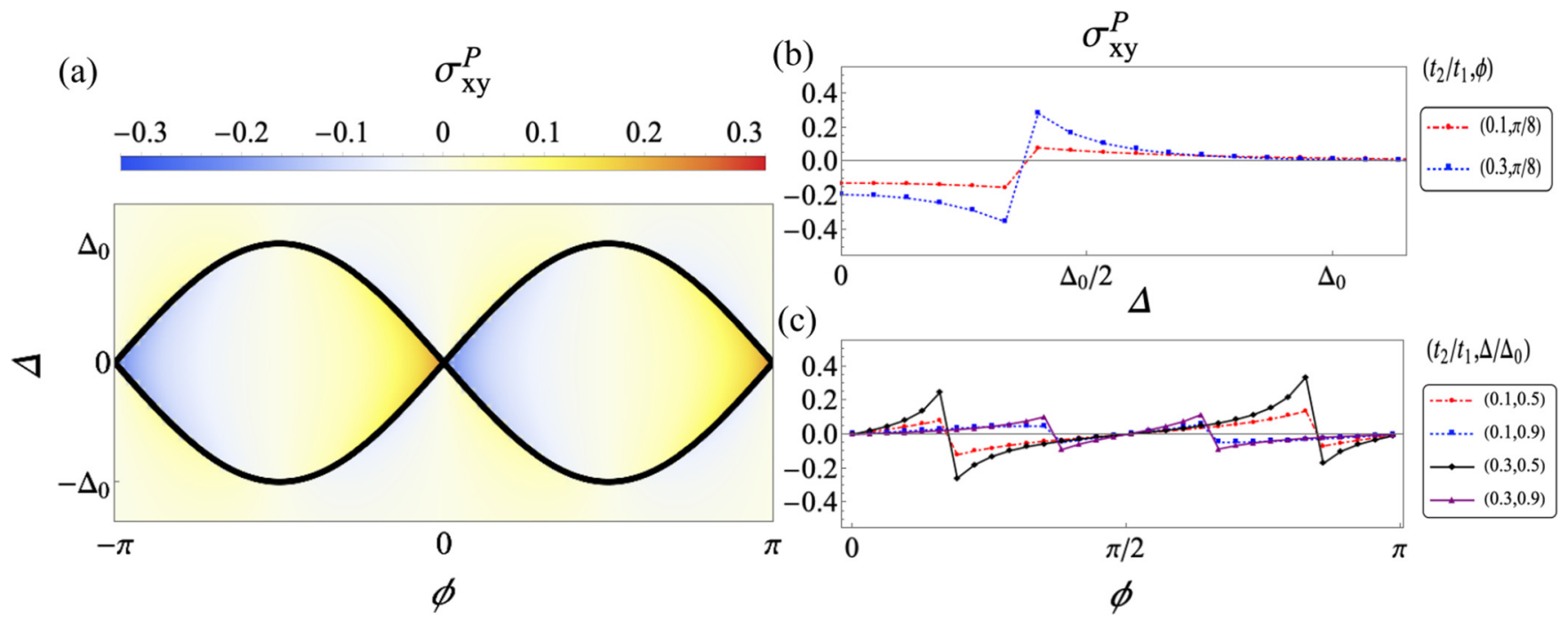

FIG. 1. (a) Transverse conductivity $\sigma_{x y}^{P}$ of the polaron in units of $g^{2} / N g_{0}^{2}$ with $g_{0}=(2 \pi a)^{2} /(3 \sqrt{3} / 2) t_{1}$, as a function of $\phi$ and $\Delta$ for $t_{2}=0.1 t_{1}$. The solid lines give the boundaries $\Delta= \pm 3^{3 / 2} t_{2} \sin \phi$ for the topological phase of the majority atoms. Panels (b) and (c) depict $\sigma_{x y}^{P}$ for fixed values of $\left(t_{2} / t_{1}, \phi\right)$ and $\left(t_{2} / t_{1}, \Delta / \Delta_{0}\right)$ respectively.

[1] D. Pimenov, A. Camacho-Guardian, N. Goldman, P. Massignan, G. M. Bruun, and M. Goldstein [to be published (2020)]. 